\title{
Inibição do crescimento micelial de Cercospora calendulae Sacc. por extratos de plantas medicinais
}

\author{
NASCIMENTO, J.M. ${ }^{1}$; SERRA, A.P. ${ }^{2 *}$; BACCHI, L.M. ${ }^{1}$; GAVASSONI, W.L. ' ; VIEIRA, M.C. ${ }^{1}$ \\ ${ }^{1}$ Universidade Federal da Grande Dourados, Pós Graduação em Agronomia - Produção Vegetal, FCA/UFGD - \\ Caixa Postal 533, CEP: 79804-970, Dourados/MS, Brasil. 'Empresa Brasileira de Pesquisa Agropecuária, Centro \\ Nacional de Pesquisa de Gado de Corte, EMBRAPA-CNPGC, Vila Popular, Caixa Postal 154, CEP: 79002-970, \\ Campo Grande/MS, Brasil. *ademar.serra@embrapa.br
}

\begin{abstract}
RESUMO: O uso de extratos e óleos essenciais de plantas medicinais tem sido amplamente estudado no controle de doenças de plantas. O objetivo da realização do presente trabalho foi avaliar o efeito de extratos de Ruta graveolens L., Mentha x villosa, Calendula officinalis L., Momordica charantia L., Symphytum officinale L., Ageratum conyzoides L. e Ricinus comunis L., nas concentrações de 0, 250, 500, 1000, 2000, 5000 e $10000 \mathrm{mg} \mathrm{L}^{-1}$, sobre a inibição do crescimento micelial de Cercospora calendulae Sacc. in vitro. Os extratos foram obtidos por infusão. O experimento foi desenvolvido no Laboratório de Fitopatologia da FCA/UFGD, estado de Mato Grosso do Sul, em delineamento inteiramente casualizado, esquema fatorial 7 extratos $x 7$ concentrações, com seis repetições. Foi detectado efeito dos extratos e suas concentrações sobre o crescimento do fungo, sendo a interação significativa. Os extratos de calêndula, arruda, hortelã e melão de São Caetano, nas maiores concentrações resultaram em maiores porcentagens de inibição, próximas de 100\%,30\%,35\% e 40\%, respectivamente, a $10000 \mathrm{mg} \mathrm{L}^{-1}$.
\end{abstract}

Palavras-chave: mancha foliar, extratos aquosos, Calendula officinalis $\mathrm{L}$.

ABSTRACT: Inhibition of the mycelial growth of Cercospora calendulae Sacc. by medicinal plants extracts. This study was carried out to investigate the effects of the extracts of Ruta graveolens L., Mentha x villosa, Calendula officinalis L., Momordica charantia L., Symphytum officinale L., Ageratum conyzoides L. and Ricinus comunis L., in the concentrations of 0, 250, $500,1000,2000,5000$ and $10000 \mathrm{mg} \mathrm{L}^{-1}$, on the inhibition of the mycelial growth of in vitro Cercospora calendulae Sacc. The extracts were obtained by infusion. The experiment was performed at the Laboratório de Fitopatologia - FCA/UFGD, in the Brazilian State of Mato Grosso do Sul. The experimental design was totally randomized, with a factorial arrangement of 7 extracts $x 7$ concentrations, with six repetitions. We detected the effect of the extracts and their concentrations on the growth of the fungus, with a significant interaction between the factor extracts and the concentration. Calendula officinalis L., Ruta graveolens L., Mentha x villosa and Momordica charantia L. extracts in higher concentration resulted in higher inhibition percentages in the order of $100 \%, 30 \%, 35 \%$ and $40 \%$, respectively.

Keywords: spot leaf, watery extracts, Calendula officinalis L.

\section{INTRODUÇÃO}

Diversas plantas medicinais têm sido testadas no controle de doenças de plantas, causadas por fungos, bactérias e nematóides, e no controle de pragas, como pulgões. A diversidade de substâncias ativas em plantas medicinais tem motivado o desenvolvimento de pesquisas envolvendo o uso de extratos e óleos essenciais, no intuito de explorar suas propriedades fungitóxicas (Franzener et al. 2003, Costa et al. 2011, Venturoso et al. 2011).
Devido à grande riqueza química das plantas medicinais que possuem princípios ativos microbiocidas, elas se tornam fontes potenciais de moléculas que podem ser empregadas na defesa de plantas contra fitopatógenos (Rodrigues et al. 2006). Plantas medicinais possuem compostos secundários que podem apresentar atividade direta, por meio de extratos brutos e óleos essenciais de plantas sobre fitopatógenos como bactérias, nematóides e fungos (Mello et al. 2006). Pesquisas realizadas in 
vitro indicam o potencial de plantas medicinais no controle de fungos fitopatogênicos pertencentes a diferentes grupos taxonômicos, como Colletotrichum graminicola, Alternaria alternata, Rhizoctonia solani, Sclerotium rolfsii e Phytophthora sp. (SchwanEstrada, 2000)

Extratos aquosos e óleos essenciais obtidos a partir de plantas medicinais (Cymbopogon martini, Thymus zygis, Cinnamomum zeylanicum e Eugenia caryophyllata)apresentam atividade antifúngica contra Botrytis cinerea (Wilson et al. 1997).

No entanto, mesmo apresentando essas propriedades antifúngicas, plantas medicinais podem ser atacadas por pragas e doenças, que poderão lhe causar prejuízo, exigindo medidas de controle para garantir sua produção. Uma questão importante é o uso de agroquímicos na produção de plantas medicinais. $\mathrm{Na}$ Europa, existem produtos registrados para o uso em melissa (Melissa officinalis L.), camomila (Matricaria chamomila L.) e menta (Mentha sp L.). Além disso, deve-se considerar que quando não se destina à indústria, essas plantas abastecem um mercado de produtos naturais que exige produção autosustentável e ecologicamente correta (Montanari Jr., 2001).

A planta de calêndula (Calendula officinalis L.) é afetada por fungos como Cercospora calendulae Sacc., que produz manchas circulares nas folhas podendo ocasionar desfolha total (Luz et al., 2001)

Dessa forma, objetivou-se avaliar o crescimento micelial de Cercospora calendulae, fungo causador de mancha foliar em plantas de calêndula, na presença de diferentes concentrações de extratos de arruda (Ruta graveolens L. ), hortelã (Mentha x villosa), calêndula (Calendula officinalis L.), melão de São Caetano (Momordica charantia L.), confrei (Symphythum officinale L.), mentrasto (Ageratum conyzoides L.) e mamona (Ricinus comunis L.).

\section{MATERIAL E MÉTODO}

O experimento foi desenvolvido no Laboratório de Fitopatologia da Faculdade de Ciências Agrárias (FCA) na Universidade Federal da Grande Dourados (UFGD) (Latitude 22014'S, Longitude $54^{\circ} 49^{\prime} \mathrm{W}$ e $452 \mathrm{~m}$ de altitude), estado de Mato Grosso do Sul, no período de julho a dezembro de 2005.

Foram testados os extratos aquosos de arruda (Ruta graveolens L.), hortelã (Mentha $\mathrm{x}$ villosa), calêndula (Calendula officinalis L.), melão de São Caetano (Momordica charantia L.), confrei (Symphythum officinale L.), mentrasto (Ageratum conyzoides L.) e mamona (Ricinus comunis L.), nas concentrações $(250,500,1.000,2.000,5.000$ e $10.000 \mathrm{mg} \mathrm{L}^{-1}$ ). Os tratamentos foram arranjados em esquema fatorial $7 \times 7$, no delineamento experimental inteiramente casualizado, com seis repetições, sendo cada repetição representada por uma placa de petri $(9 \mathrm{~cm}$ de diâmetro) contendo BDA solidificado e discos de micélio do patógeno.

O isolado de Cercospora calendulae Sacc. foi obtido na micoteca do Laboratório de Fitopatologia. Para a obtenção dos extratos, a parte aérea das espécies das plantas medicinais avaliadas foi coletada em junho/julho de 2005 , no Horto de Plantas Medicinais (HPM) na UFGD. A identificação botânica foi realizada pela professora Dra Maria do Carmo Vieira da FCA/UFGD. As exsicatas estão depositadas no herbário da UFGD, Dourados-MS.

O material coletado foi secado ao ar ambiente por 10 dias. Os extratos foram obtidos segundo adaptação da metodologia utilizada por Ferris \& Zheng (1999), obtidos por infusão da matéria seca em água destilada aquecida em forno de microondas $\left(95^{\circ} \mathrm{C}\right)$, onde misturou-se $10 \mathrm{~g}$ de massa da matéria seca com $100 \mathrm{~g}$ de água destilada. A mistura foi mantida em repouso por 24 horas e filtrada em papel de filtro. Posteriormente, foram esterilizados através de tindalização, permanecendo a $100^{\circ} \mathrm{C}$ por uma hora sendo este procedimento repetido por três dias consecutivos.

Para se avaliar o efeito de extratos das espécies avaliadas sobre o desenvolvimento de Cercospora calendulae, estes foram adicionados em meio de cultura BDA (batata-dextrose-ágar) fundente, de modo a se obter as respectivas concentrações conforme metodologia descrita por Barros et al. (1995). Como testemunha foram utilizadas placas contendo apenas meio de cultura BDA, vertidos em placas de petri ( $9 \mathrm{~cm}$ de diâmetro), e depois de sua solidificação, os discos de micélio do patógeno, de $2 \mathrm{~mm}$ de diâmetro e 14 dias de idade, foram transferidos para o centro das placas. Essas foram vedadas com filme plástico, e incubadas a uma temperatura de $25^{\circ} \mathrm{C}$.

As avaliações foram realizadas diariamente, entre 17 e 19 dias após repicagem, no do momento em que as colônias atingissem $3 / 4$ da superfície da placa na testemunha (Stangarlin et al., 1999).

Foi avaliado o crescimento do micélio, medindo-se o diâmetro da colônia fúngica, em dois sentidos opostos. A partir dos resultados obtidos determinou-se a percentagem de inibição do crescimento micelial (PIC) e a taxa de crescimento (Tx), por meio das fórmulas apresentadas a seguir:

$$
\begin{aligned}
& \mathrm{PIC}=\left(\frac{\text { diâmetro da testemunha }- \text { diâmetro do tratamento }}{\text { diâmetro da testemunha }}\right) \cdot 100 \\
& \mathrm{Tx}=\frac{\text { diâmetro final da colônia }}{\text { número de dias de incubação }} .100
\end{aligned}
$$


Os dados foram submetidos à análise de variância pelo teste $F$, e para o caso de diferenças significativas entre os tratamentos, foi realizada a análise de regressão simples para o fator concentração e teste de Tukey para as médias de extratos, utilizando-se o aplicativo computacional SAEG 9.1.

\section{RESULTADO E DISCUSSÃO}

Detectou-se o efeito dos extratos de plantas e suas concentrações sobre a taxa de crescimento e a porcentagem de inibição de Cercospora calendulae, sendo a interação significativa $(p<$ $0,01)$. Dessa forma ajustaram-se equações de regressão aos níveis do fator concentração de cada extrato.

Para a taxa de crescimento não houve efeito das concentrações para os extratos de hortelã, confrei, mentrasto e mamona (Figura $1 \mathrm{~A} \mathrm{e}$ B), no entanto, a aplicação dos extratos de arruda, calêndula e melão de São Caetano nas maiores concentrações reduziram significativamente a taxa de crescimento. À medida que a taxa de crescimento reduziu, automaticamente aumentou a porcentagem de inibição, para todos os extratos (Figura $1 \mathrm{~A}$ e $\mathrm{B}$, 2A e B).

Para os extratos de arruda, hortelã, calêndula e melão de São Caetano, houve maior porcentagem de inibição de crescimento micelial à medida que se elevou a concentração. $O$ extrato de calêndula na concentração de $10000 \mathrm{mg} \mathrm{L}^{-1}$ inibiu $98,67 \%$ o crescimento micelial, sendo a DL50 de $6361 \mathrm{mg} \mathrm{L}^{-1}$ (Tabela 1 e Figura 2A e B). Para os outros extratos, embora com efeito sobre o crescimento micelial do fungo fitopatogênico, na maior concentração estudada, não se atingiu 50\% de inibição.

Nas maiores concentrações (5000 e 10000 $\left.\mathrm{mg} \mathrm{L}^{-1}\right)$, o extrato de calêndula foi significativamente mais eficiente na inibição do cescimento micelial, diferindo de todos os demais, seguido dos extratos
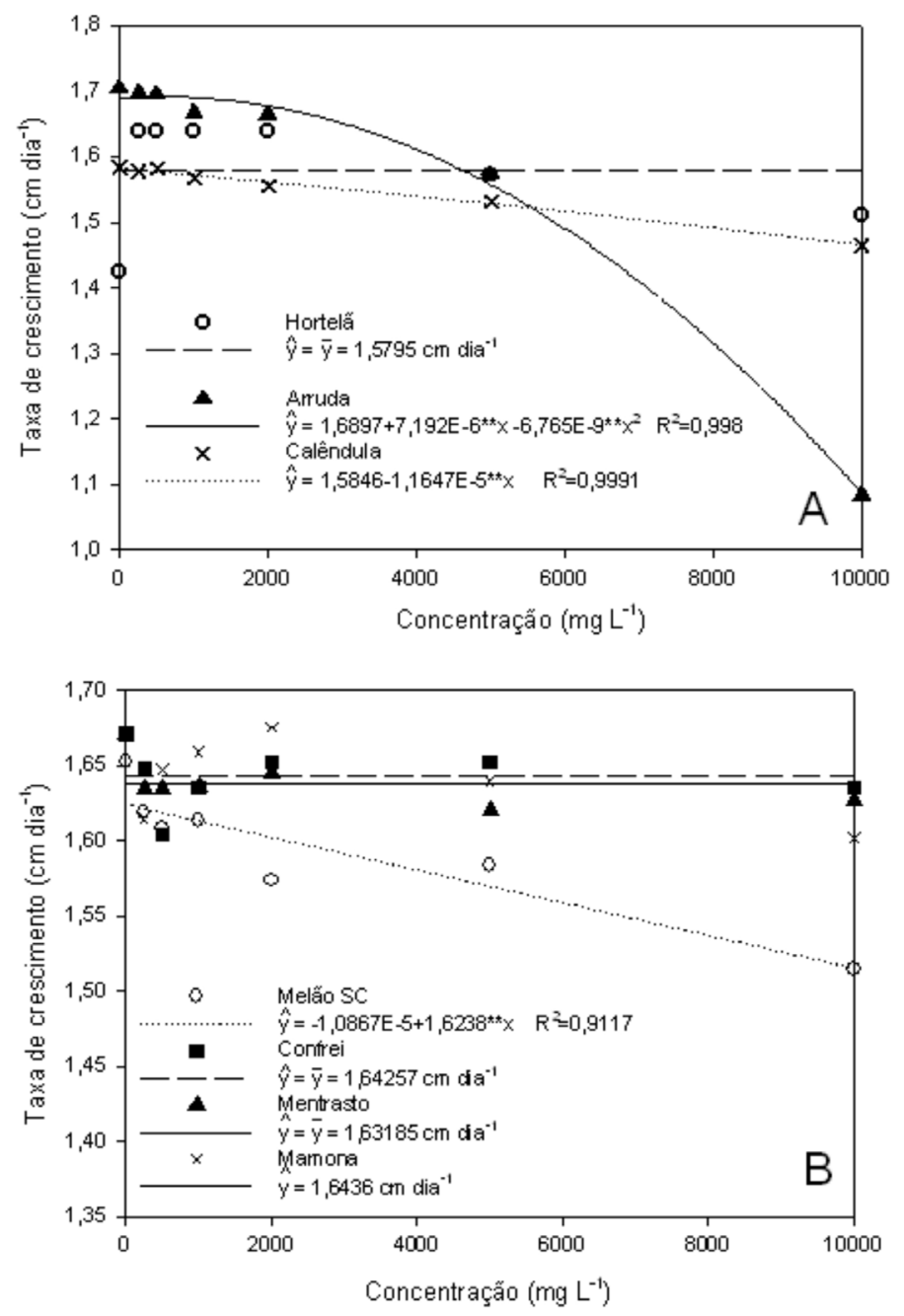

Figura 1A e 1B. Taxa de crescimento micelial $\left(\mathrm{cm} \mathrm{dia}^{-1}\right)$ de $C$. calendulae para os extratos de arruda, hortelã, calêndula, melão de São Caetano, confrei, mentrasto e mamona. 
de arruda, hortelã e melão de São Caetano. A menor inibição nestas concentrações foi observada com a utilização do extrato de confrei. Em menores concentrações, os extratos de confrei (500 $\left.\mathrm{mg} \mathrm{L}^{-1}\right)$ e mamona (250 $\left.\mathrm{mg} \mathrm{L}^{-1}\right)$ apresentaram algum efeito sobre o fungo, porém este efeito não foi em função da concentração, como observado na Figura 2B, e ficaram bem abaixo da inibição máxima obtida com os extratos de calêndula $(98,67 \%)$, hortelã $(34,97 \%)$ e melão de São Caetano $(37,85 \%)$ (Tabela 1$)$.

O efeito antifúngico de calêndula foi observado por outros autores. Trabalho realizado com óleo essencial de calêndula extraído das flores, mostrou que houve supressão na atividade antifúngica de 23 espécies de Candida sp (Gazim, et al. 2008).

O efeito de inibição parcial do crescimento micelial, observado para os extratos de melão de São Caetano, hortelã e arruda também é relatado por outros autores. Os resultados variam em função da espécie fúngica estudada. Celoto et al. (2008), trabalhando com porcentagem de inibição de crescimento micelial de Colletotricum gloesporioides (agente causador da antracnose no mamoeiro) observaram que o extrato aquoso de melão de São Caetano proporcionou mais de $90 \%$ de porcentagem de inibição, diferindo significativamente dos demais extratos, sendo que o extrato aquoso de arruda, incorporado (20\%) em BDA, apresentou porcentagem de inibição de $5 \%$. Por outro lado, Salvadori et al. (2003) relatam que extrato bruto das folhas de arruda, incorporado a $25 \%$ em BDA e autoclavado, apresentou $23,5 \%$ de inibição de crescimento micelial de $C$. gloeosporioides.

Em trabalhos desenvolvidos por Ribeiro \& Bedendo (1999), os extratos aquosos de mamona e hortelã, dentro dos limites de 200 a $10000 \mathrm{mg} \mathrm{L}^{-1}$, promoveram a inibição relativa do desenvolvimento de micélio de Colletotricum gloesporioides. Esta inibição foi diretamente proporcional às concentrações utilizadas. No caso de Cercospora calendulae, não foi observado efeito, sobre a taxa de inibição do crescimento micelial, dependente da concentração para o extrato de mamona (Figura 2B).

Schwan-Estrada \& Stangarlin (2001) citam que arruda (Ruta graveolens L.), alho (Allium sativum L.) e mentrasto (Ageratum conyzoides L.) têm sido utilizadas para estudos de inibição de crescimento micelial e esporulação de fungos fitopatogênicos in vitro (Rhizoctonia solani, Sclerotium rolfsii, Alternaria alternata, Phytophthora sp e Colletotrichum graminicola).

Estudando extratos vegetais no controle de fungos fitopatogênicos a soja, Venturoso et al. (2011) observaram que houve diferenças significativas em relação à testemunha para crescimento micelial de Cercospora kikuchii, com extratos de hortelã, arruda e melão de São Caetano, na concentração de $20 \%$.

\section{CONCLUSÂO}

Os extratos aquosos de calêndula, hortelã, arruda, e melão de São Caetano, na maior concentração testada (10000 mg L-1), inibiram o crescimento de Cercospora calendulae na ordem de $100 \%, 30 \%, 35 \%$ e $40 \%$, respectivamente.

Estudos subsequentes devem ser realizados in vivo visando a confirmação do efeito inibitório desses extratos sobre $C$. calendulae, através da observação do sintoma da doença na planta.

TABELA 1. Efeito de extratos de plantas medicinais com diferentes concentrações $\left(\mathrm{mg} \mathrm{L}^{-1}\right)$ sobre a porcentagem de inibição de C. calendulae.

\begin{tabular}{ccccccc}
\hline Tratamentos & \multicolumn{7}{c}{ Concentrações $\left(\mathbf{m g ~ L}^{-1}\right)$} \\
\cline { 2 - 7 } & $\mathbf{2 5 0}$ & $\mathbf{5 0 0}$ & $\mathbf{1 0 0 0}$ & $\mathbf{2 0 0 0}$ & $\mathbf{5 0 0 0}$ & $\mathbf{1 0 0 0 0}$ \\
\hline Arruda & $0,29 \mathrm{~b}$ & $4,93 \mathrm{ab}$ & $0,85 \mathrm{ab}$ & $13,00 \mathrm{a}$ & $10,19 \mathrm{bc}$ & $29,86 \mathrm{bc}$ \\
Hortelã & $0,00 \mathrm{~b}$ & $0,00 \mathrm{~b}$ & $0,00 \mathrm{~b}$ & $0,00 \mathrm{~d}$ & $19,40 \mathrm{~b}$ & $34,97 \mathrm{~b}$ \\
Calêndula & $2,88 \mathrm{~b}$ & $3,56 \mathrm{~b}$ & $10,89 \mathrm{a}$ & $11,77 \mathrm{ab}$ & $34,44 \mathrm{a}$ & $98,67 \mathrm{a}$ \\
Melão S.C & $3,13 \mathrm{~b}$ & $2,26 \mathrm{~b}$ & $7,34 \mathrm{ab}$ & $11,02 \mathrm{abc}$ & $18,22 \mathrm{~b}$ & $37,85 \mathrm{~b}$ \\
Confrei & $3,36 \mathrm{~b}$ & $15,03 \mathrm{a}$ & $6,99 \mathrm{ab}$ & $1,96 \mathrm{bcd}$ & $1,96 \mathrm{c}$ & $6,62 \mathrm{e}$ \\
Mentrasto & $9,90 \mathrm{ab}$ & $9,89 \mathrm{ab}$ & $9,43 \mathrm{ab}$ & $6,63 \mathrm{abcd}$ & $13,63 \mathrm{~b}$ & $12,00 \mathrm{de}$ \\
\hline Mamona & $18,01 \mathrm{a}$ & $9,70 \mathrm{ab}$ & $6,11 \mathrm{ab}$ & $1,62 \mathrm{~cd}$ & $11,72 \mathrm{bc}$ & $21,83 \mathrm{~cd}$ \\
\hline
\end{tabular}

Nas colunas, médias seguidas pela mesma letra, não difere significativamente de acordo com o teste Tukey a $5 \%$ de probabilidade. 

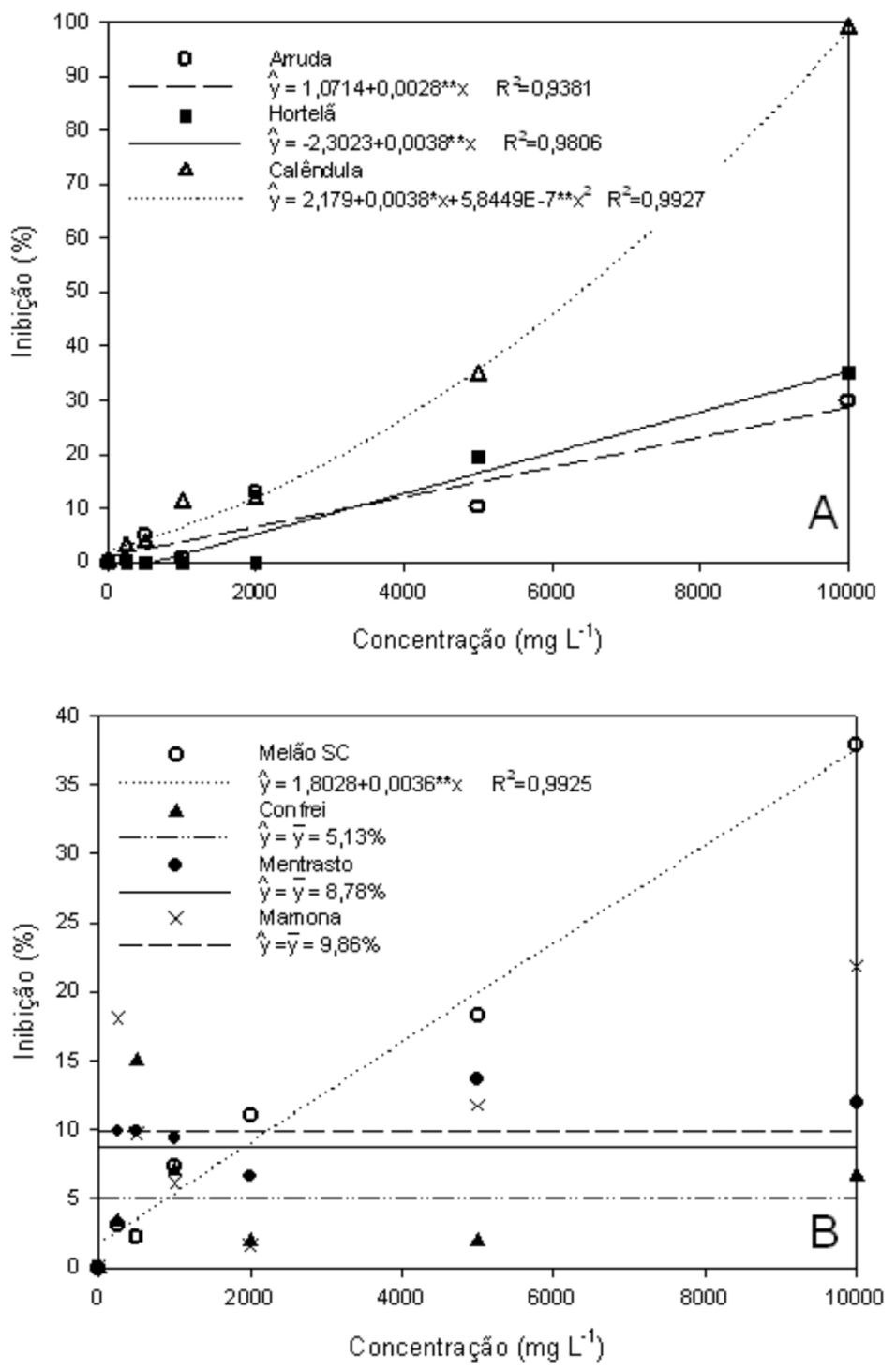

FIGURA 2A E 2B. Porcentagem de inibição de crescimento micelial de C. calendulae para os extratos aquosos de arruda, hortelã, calêndula, melão de São Caetano, confrei, mentrasto e mamona.

\section{REFERÊNCIA}

BARROS, S.T. et al. Efeito de extrato de alho (Allium sativum) sobre o crescimento micelial e germinação de conídios de Curvularia spp. e Alternaria spp. Summa Phytopathologica, v.21, n.2, p.168-70, 1995.

CELOTO, M. et al. Atividade antifúngica de extratos de plantas a Colletotrichum gloesporioides. Acta Scientiarum, v.30, n.1, p.1-5, 2008.

COSTA, A.R.T. et al. Ação do óleo essencial de Syzygium aromaticum (L.) Merr. \& L.M. Perry sobre as hifas de alguns fungos fitopatogênicos. Revista Brasileira de Plantas Medicinais, v. 13, n.2, p.240-245, 2011.

FERRIS, H. \& ZHENG, L. Plant sources of Chinese herbal remedies: effects on Pratylenchus vulnus and Meloidogyne javanica. Journal of Nematology, v.31, n.3, p.241-63, 1999.
FRANZENER, G. et al. Atividade antifungica e indução de resistência em trigo a Bipolaris sorokiniana por Artemisia camphorata. Acta Scientiarum, v.25, n.2, p.503-7, 2003.

GAZIM, Z.C. et al. Antifungal activity of the essential oil from Calendula officinalis L. (asteraceae) growing in Brazil. Brazilian Journal of Microbiology, v.39, n.1, p.61-3, 2008.

LUZ, L.A. et al. Instructivo técnico de Calêndula officinalis. Revista Cubana de Plantas Medicinais, v.1, n.1, p.23-7, 2001.

MELLO, A.F.S. et al. Potencial de controle de ervade-santa-maria sobre Pratylenchus brachyurus. Fitopatologia Brasileira, v.31, n.5, p.513-6, 2006.

MONTANARI J.R. Aspectos legais da produção comercial de plantas medicinais. Agroecologia, v.6, n.1, p.25-6, 2001. 
RIBEIRO, L.F \& BENDENO, I.P. Efeito inibitório de extratos vegetais sobre Colletotrichum gloeosporioides - agente causal da podridão em frutos de mamoeiro. Scientia Agrícola, v.56, n.4, p.1267-71, 1999.

RODRIGUES, E. et al. Avaliação de atividade antifúngica de extratos de gengibre e eucalipto in vitro e em fibras de bananeira infectadas com Helmintosporium sp. Acta Scientiarum, v.28, n.5, p.123-7, 2006.

SALVADORI, R.K. et al. Atividade antifúngica dos extratos brutos de Corymbia citriodora, Cymbopogon citratus, Ruta graveolens e Curcuma longa. Fitopatologia Brasileira, v.28, n.2, p.360-1, 2003.

SCHWAN-ESTRADA, K.R.F. et al. Uso de extratos vegetais no controle de fungos fitopatogênicos.

Revista Floresta, v.30, n.1, p.129-37, 2000.
SCHWAN-ESTRADA, K.R.F. \& STANGARLIN, J.R. Plantas medicinais mo controle fitossanitário. In: jornada paulista de plantas medicinais. Natureza, ciência e comunidades, 5, 2001, Botucatu. Anais... Botucatu: UNESP, 2001. p.43-8.

STANGARLIN, J.R. et al. Plantas medicinais e controle alternativo de fitopatógenos. Biotecnologia Ciência \& Desenvolvimento, v.2, n.11, p.16-21, 1999.

VENTUROSO, L. et al. Atividade antifúngica de extratos vegetais sobre o desenvolvimento de fitopatógenos. Summa Phytopathologica, v.37, n.1, p.18-23, 2011.

WILSON, C. L. et al. Rapid evaluation of plant extracts and essential oils for antifungal activity agaist Botrytis cinerea. Plant Disease, v.18, n.2, p.204-10, 1997. 